\title{
Research Square \\ Improving the Quality of Routing Service using Metaheuristic PSO algorithm in VANET Networks
}

\section{Amir Javadpour}

Guangzhou University

\section{Samira Rezaei}

University of Groningen: Rijksuniversiteit Groningen

Arun Kumar Sangaiah ( $\nabla$ arunkumarsangaiah@gmail.com )

VIT University, Vellore https://orcid.org/0000-0002-0229-2460

\section{Adam Slowik}

Koszalin University of Technology: Politechnika Koszalinska

Shadi Mahmoodi Khaniabadi

Universiti Sains Malaysia

\section{Research Article}

Keywords: Vehicular Ad-hoc Networks, QoSR, QoS, VANETs, PSO Algorithm, VanetMobisim

Posted Date: April 27th, 2021

DOI: https://doi.org/10.21203/rs.3.rs-449311/v1

License: (c) (1) This work is licensed under a Creative Commons Attribution 4.0 International License. Read Full License 


\title{
Improving the Quality of Routing Service using Metaheuristic PSO algorithm in VANET Networks
}

\author{
Amir Javadpour ${ }^{1}$, Samira Rezaei ${ }^{2}$, Arun Kumar Sangaiah ${ }^{3,4}$, Adam Slowik ${ }^{5}$, Shadi Mahmoodi Khaniabadi ${ }^{6}$ \\ ${ }^{1}$ School of Computer Science and Technology, Guangzhou University, Guangzhou, China, 510006. (a javadpour@e.gzhu.edu.cn) \\ ${ }^{2}$ Bernoulli Institute for Mathematics and Computer Science, University of Groningen, The Netherlands. (rezaei.samiraa@gmail.com) \\ ${ }^{3}$ School of Computing Science and Engineering, VIT University, Vellore, India. (arunkumarsangaiah@gmail.com) \\ ${ }^{4}$ Department of Industrial Engineering and Management, National Yunlin University of Science and Technology, Taiwan \\ ${ }^{5}$ Department of Electronics and Computer Science, Koszalin University of Technology, Poland. (adamslowik@tu.koszalin.pl) \\ ${ }^{6}$ Department of Computer Science and Engineering, USM Engineering Campus, Pulau Pinang, Malaysia, 14300. (shadi.mahmoodi164@gmail.com)
}

\begin{abstract}
VANETs are organized to progress road protection with no specific need for any fixed infrastructure. Subsequently, the movement of all vehicles can be planned in the upcoming future, based on perceived information, Quality of Services Routing (QoSR) algorithms can be pressured on its available options, paths, and links and according to criteria and reliability of the QoSR. Awareness of QoSR to the environmental conditions of the network of vehicles, such as the location of vehicles, direction and speed that can be obtained .This study is to reduce the effects of unpredictable problems on the best pathway to replace the broken path / link. In this article, A QoSR with Particle Swarm Optimization (QoSR-PSO) for improving QoSs in vehicular ad-hoc networks has been used. The particle swarm optimization algorithm by modeling the behavior of a set of particles looks for the optimal solution of the problem. In order to perform simulation experiments, NS2 simulator and VanetMobisim have been used. The comparison results with benchmark studies show the improvement in packet delivery rate (PDR), delay, Packet Drop and overload.
\end{abstract}

Keywords: Vehicular Ad-hoc Networks, QoSR, QoS, VANETs, PSO Algorithm, VanetMobisim.

\section{Introduction}

The VANETs and Cellular Mobile Networks (CMN) have no previous defined topology and nodes can move freely and changes the topology. Nodes can be considered as routers or end-systems to share communication resources with others and transmit data through dynamic backbone. The VANETs are a different form of MANETs with some differences in communication nodes and environment [1], [2]. WAVE (Wireless Access in Vehicular Environments) protocol provides basic conditions for implementing VANETs in Dedicated ShortRange Communications (DSRC). And DataPacket transformation involves sending packets between the vehicles and from a vehicles to RoadSideUnits (RSU)[1], [2].

Rapid advances in the field of wireless communications technologies have created a lot of changes in various areas in our everyday lives [3]. One of the applications which is expected to be very lucrative is the safety of vehicles. Smart vehicles with wireless communication equipment can quickly communicate with each other and with RSUs [4]. It helps to prevent traffic congestions, suggest alternative routes, estimate arrival times and generally improve road safety and performance [6], [7]. Discussions and investigation on the causes of accidents have increased not only by researches in universities but even by industry and governments.

Routing in VANETs is affected by several conditions such as environment, vehicles location, directions and speed and atmospheric conditions. In addition, drivers behavior regarding their tendency to increase / decrease acceleration is besides an main parameter that the QoSR should be considered of them [8], [9]. An important issue in this study is to reduce the random things of problems on a best pathway or replace the broken path / link. This task can be performed by exploring valid paths during the routing process. In the proposed method, we use PSO algorithms to find the suitable route in VANETs. The important issues of VANETs is to maintain QoS in traffic jams. On the other hand, driver behavior; restrictions and road rules; mobility and high speeds cause changes in topology of the network. In this paper, we have presented a quality improvement structure using particle swarm optimization (PSO) in VANETs. It combines IEEE 802.11P-based multi-hub clustering through the objective of accomplishing HighPacketDeliveryRates (HPDR) and low latency, although the minimum tradition of infrastructure. The idea is based on reducing the Clusters. The structure of paper is as follows; the first section contains the introduction of VANETs and QoSR, and then we will review some related research background. The next section presents the proposed method contains of QoSR and communication methods; and 
for implementation common standards and simulation tools are considered. In forth section the results of applying NS2 simulator to evaluate the proposed method are presented. We also used VanetMobiSim [10]; a specific simulator for VANETs to examine the behavior of vehicles. And the next section we summarized our work with discussions and conclusions.

\section{Literature}

Despite several presented routing methods for VANETs, high mobility of high speed cars and different speeds in different environments make a lot of them not efficient enough. Supporting the quality of service in data transmission plays a very important character in the Intellectual Transport Structure (ITS). In recent years, routing algorithms have been considered in VANETs. However, given a specific features of VANETs such as Speed, having enough response time and so on, proposed algorithms for wireless are not appropriate for VANETs that are based on data dissemination with dynamic spine. Therefore, providing new routing methods for VANETs should be specially designed [6]. The network should be designed in a way which transmit delay sensitive messages such as safety messages instantly. Safety messages include post-accident warning, road safety warning, speed/stop advisor, road traffic warning, line change and emergency vehicle approach. Information dissemination involves various protocols for transmitting information that are used dependent on category of APPs of the message. Flooding dissemination and geographic distribution, request-response, subscription, broadcast and routing are all types of data transfer protocols that have been used. Routing in VANET is used to optimally disseminate, reduce overhead and reduce the delivery latency of a message.

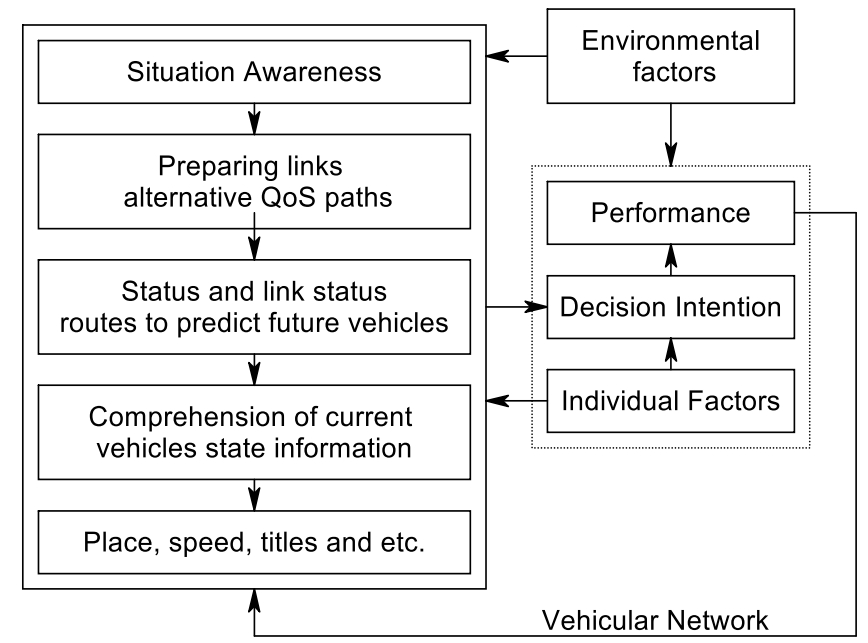

Fig. 1. Classical model for Routing in VANETs [4].

Authors in [4] present a situation aware (SA) routing protocol implementing ant colony algorithm. The purpose of this protocol is to resolve the path locally and send error messages to the source node to interrupt the current data transfer. The conceptual model of this article is illustrated in Figure 1. First step in SA refers to awareness of the environmental and vehicles conditions such as the location, direction, speeds, traffic and atmospheric conditions. In the next step, all the information should be used to present an up to date routing algorithm. Finally, in last step it is important to prediction the coming position of the VANETs and expect the connection period and its QoSR Achieved Packet Delivery Ratio has 7.65\% error.

In [13] MPLS-based routing algorithm is proposed for VANETs. It assumes that vehicle has a numerical map using destination also source addresses to find the best route. Authors in [14] presented an approach in which each node has a weight. Weights are calculated using connection quality, mobility and so on. Therefore, the algorithm can increase the reliability. A MtiHop (MH) solutions is used in [15] which procedures collective density and MPLS in multi hop as a criterion for routing nodes. This criterion is calculated and aggregated using beacon delay messages in each node (calculated and aggregated for each multi hop neighbors). The result is sent to all neighbors and eventually the node thru inferior collective density is selected as ClusterHead. The MultiHop algorithm in [16] tries to follow pattern of moving nodes and increases the survival time of the cluster and reduces global overhead. In this algorithm, the vehicle's destination is an important criterion for routing and uses current location criteria, temporal and final speed and destination for routing. Of course, it may be difficult to get the final destination. Cluster size changes according to the crowdedness of vehicles, the speed and minimum required bandwidth or QoS that this minimum required bandwidth can be predefined. In addition to the above divisions, based on the routing method, the network can be separated three groups. In proactive routing (1), data routing takes place before any need to data transmission while in the reactive (2) method routing is done on demand. When in a network, the location of the nodes is quite clear, the use of the proactive method is reasonable because the reactive 
methods require a lot of energy to get the path. In the hybrid mode(3), an intermediate state is used between these two methods, as at the beginning of the operation of the network, there was primary network information for the nodes and during the network operation, the paths are updated with the information that the nodes collect from the network [17]. The details of our method and others have been investigated in Table 1.

Table 1 - Comparing different routing algorithms in VANET.

\begin{tabular}{|c|c|c|}
\hline Routing algorithms & Advantages & Disadvantages \\
\hline SAMQ [4] & $\begin{array}{l}\text { This method is proposed for MultiLimit routing and } \\
\text { has a high permittivity. In addition, delayed packet } \\
\text { sending is appropriate from the vehicle to the base } \\
\text { station. } \\
\text { High QoSR, Low Delay, High Throughput and PDR. }\end{array}$ & $\begin{array}{l}\text { When the link failure occurs, the middle node cannot } \\
\text { change the path / link but sends the MSG to leading } \\
\text { node to change to the BackupPath. During this time, } \\
\text { data transfer will stop since the target is unavailable. } \\
\text { High QoSR, Low Delay, High Throughput and PDR. }\end{array}$ \\
\hline FIOODING [18] & $\begin{array}{l}\text { By changing the network topology, it adapts quickly. } \\
\text { High QoSR, Low Delay. }\end{array}$ & $\begin{array}{l}\text { Due to the repetition and replication of data packets, } \\
\text { it creates a high traffic, overlapping of vehicular ad } \\
\text { hoc networks, decreasing life time }\end{array}$ \\
\hline SPIN [19] & $\begin{array}{l}\text { Reducing energy consumption and using the CSMA } \\
\text { protocol to ensure delivery of packets and reaching the } \\
\text { destination. } \\
\text { High QoSR, High PDR. }\end{array}$ & $\begin{array}{l}\text { Computational complexity and re-establishment of } \\
\text { route reconstruction }\end{array}$ \\
\hline Genetic Algorithm [20] & $\begin{array}{l}\text { Overhead reduction, optimal permittivity. } \\
\text { High QoSR, Low Delay }\end{array}$ & $\begin{array}{l}\text { The problem of coordination between the RSU in a } \\
\text { range that there is prevention }\end{array}$ \\
\hline MPLS-based [13] & $\begin{array}{l}\text { Using MLPS and destination address (DS) and source } \\
\text { address (SA) can calculate the route. } \\
\text { High QoSR, Low Delay }\end{array}$ & $\begin{array}{l}\text { Computational complexity and re-establishment of } \\
\text { route reconstruction in high traffic. }\end{array}$ \\
\hline PUMA algorithm [14] & $\begin{array}{l}\text { High quality and good mobility } \\
\text { High QoSR, Low Delay, High Throughput and PDR. }\end{array}$ & $\begin{array}{l}\text { The weight of each node is calculated by using } \\
\text { connection quality and its makes Computational } \\
\text { complexity }\end{array}$ \\
\hline Multi hop [16] & $\begin{array}{l}\text { Collective density and MPLS in multi hop as a } \\
\text { criterion for routing nodes, criterion is calculated and } \\
\text { aggregated by using the delay of beacon messages. } \\
\text { Each node is sent to each of the neighbors and } \\
\text { eventually the node is selected as the head of the } \\
\text { cluster which has a lower collective density. }\end{array}$ & $\begin{array}{l}\text { nodes moving and increases the survival time of the } \\
\text { cluster and reduces global overhead }\end{array}$ \\
\hline
\end{tabular}

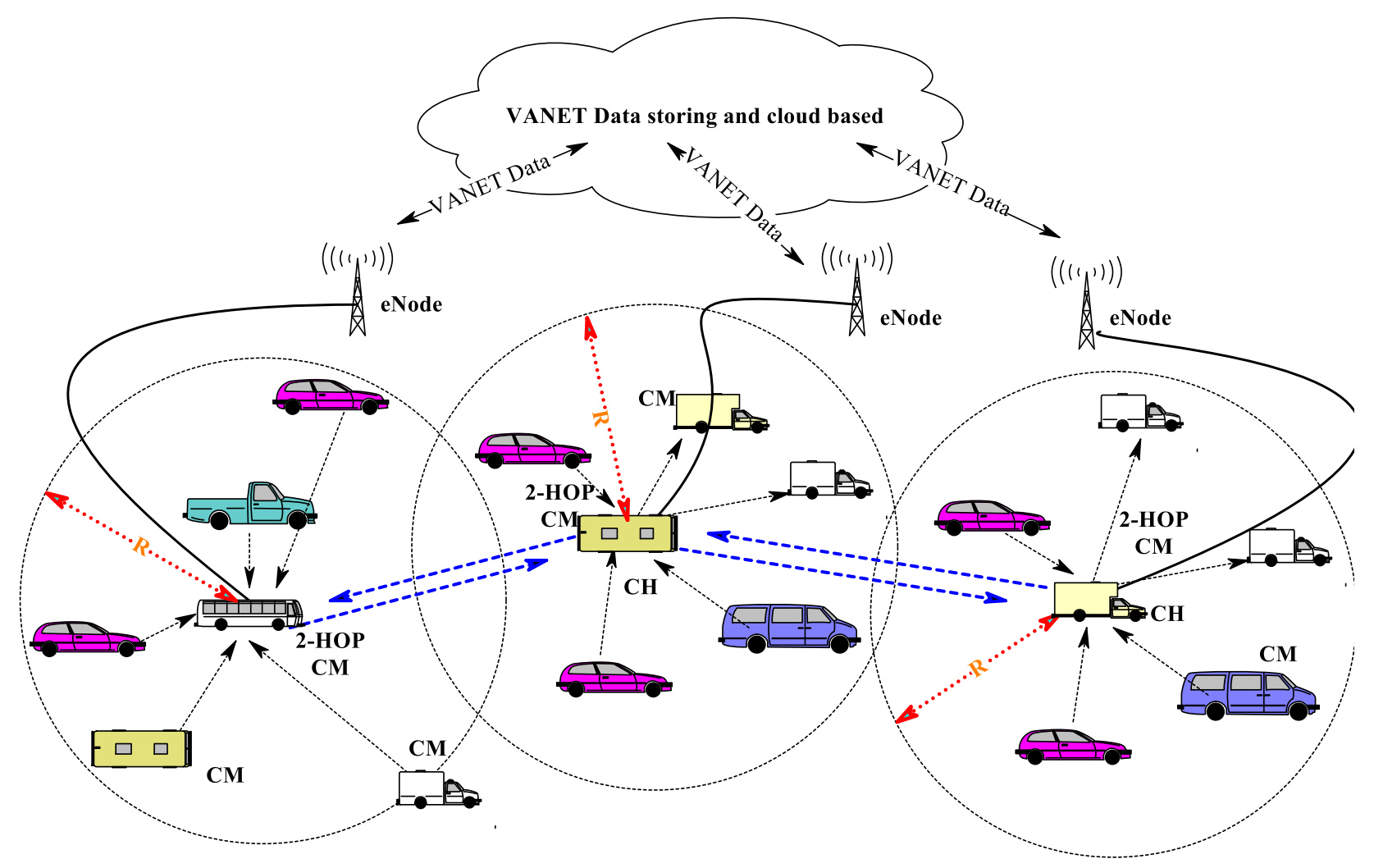

Fig 2. An overview of node communication in VANETs[21]. 


\section{Proposed method (QoSR-PSO)}

In VANETs, several nodes act as hosts and routers at the same time. Due to movement of them in different topologies, the major challenges of mobile ad hoc network are to create reliable paths and reduce latency[22]. Nodes may also run out of energy and break down the network. It is important to consider energy level of nodes[4]. In this section, a QoSR is proposed for VANETs to reduce response time, improving QoS, updating the load balancing by using the PSO algorithm. Recently, due to the large development of heuristic algorithms for various applications, many researchers have focused on using these algorithms for routing in the network. The major disadvantages of these methods are uncertainty in reaching the optimal solution. The most important advantages are the abundance of maneuverability, their simple and very fast design. Choosing the right fitness function is necessary to choose the best cluster as there are many cases in which sender and receiver are not directly connected and they need to find the best routing path.

In the proposed communication model of nodes and RSUs is a bilateral graph. Vehicles in the transition domain of a cluster head $(\mathrm{CH})$ is shown with dashed line. Cluster members $(\mathrm{CM})$ communicate directly with their respective $\mathrm{CHs}$ [21]. CMs can transfer with others through IEEE80211P MAC. eNode is a sophisticated base station that controls radio communications by many tools and integrates radio resource management and decision making. When an eNodeBS receives a data packet for propagation, it will be distributed multiple times for all CHs covered by RSUs.

This section describes in detail the proposed algorithm based on particle swarm optimization to improve reliability. PSO is a soft computational method that is generally based on bird swarm theory. PSO development is based on continuous optimization of nonlinear functions. It searches for space and optimal positioning, adjusts the speed of each vehicle on the vehicle network, taking into account the vehicle's previous best position and the neighbor's best previous locations. Selecting the next vehicle $(\mathrm{CH})$ is the most important responsibilities in VANETs because a number of constraints such as frequent topological changes, interference, predetermined transport direction, clustering, very high vehicle speeds, etc. are involved in VANETs.

We present our QoSR routing algorithm based on PSO. It is a global optimization method that solves problems when the answer is an argument or a external in $\mathrm{n}$ dimensional area. PSO searches for optimal solution of the problem by modeling the behavior of a set of particles. By moving these particles in the reaction space, the evaluation of the results is performed on the basis of a merit criterion. Over time, particles tend to accelerate towards particles with a higher merit values in the same communication group. The abundance of swarming particles makes the method flexible against the local optimal response problem. It specifies any particle that has a position.

$$
\begin{aligned}
& v_{i}^{t+1}=w^{*} v_{i}^{t}+c_{\mathrm{i}}^{*} \operatorname{rand}_{1} *\left(y_{i}-x_{i}^{t}\right)+c_{j}^{*} \text { rand }_{2} *\left(y_{j}-x_{i}^{t}\right) \\
& x_{i}^{t+1}=x_{i}^{t}+v_{i}^{t+1} \\
& w=w_{\text {max }}-t^{*} \frac{w_{\text {max }}-w_{\text {min }}}{t_{-} \text {max }}
\end{aligned}
$$

Particle i move in space with speed of $\mathrm{v}_{\mathrm{i}}{ }^{(t)}$ at iteration t. Particles memorize the best positions throughout their lives. The best experience of a particle or the best position observed by particle is called $\mathrm{y}_{\mathrm{i}}$. Particles are aware of the best observed position in the entire group which is called $\hat{y}$. The $c_{i}$ and $c_{j}$ are constant and $w$ is the initial weight that decreases linearly from $W_{\max }$ to $\mathrm{W}_{\min }$. $t$-max is the maximum number of repetitions that are considered for the algorithm. It is expected to increase the QoS by utilizing PSO in dedicating the communication path between two vehicles. The flowchart of applying PSO to VANET is illustrated in Figure 3. The details of our method for path routing is shows in Table 2. 
Table 2 - QoSR-PSO path routing optimization algorithm.

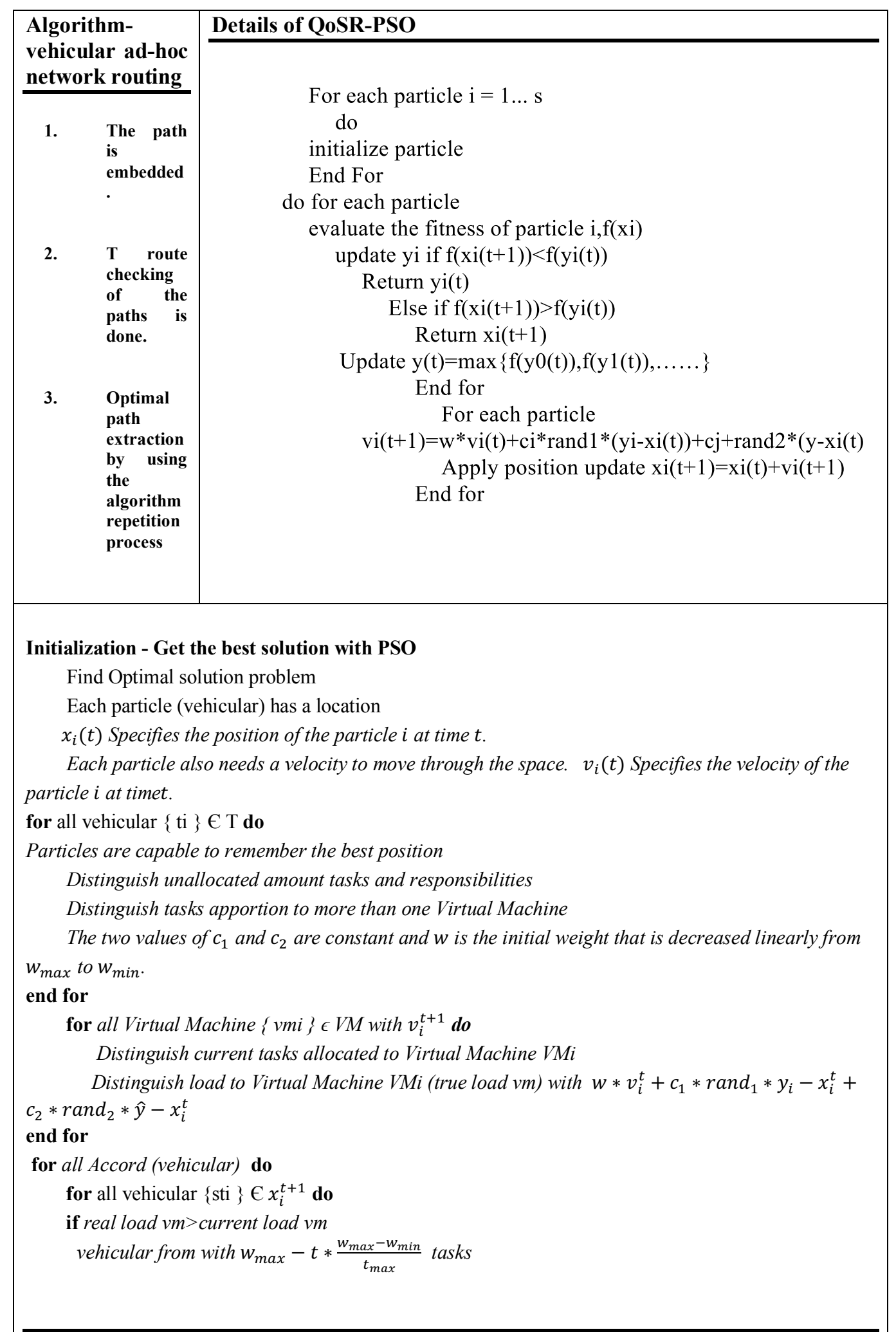




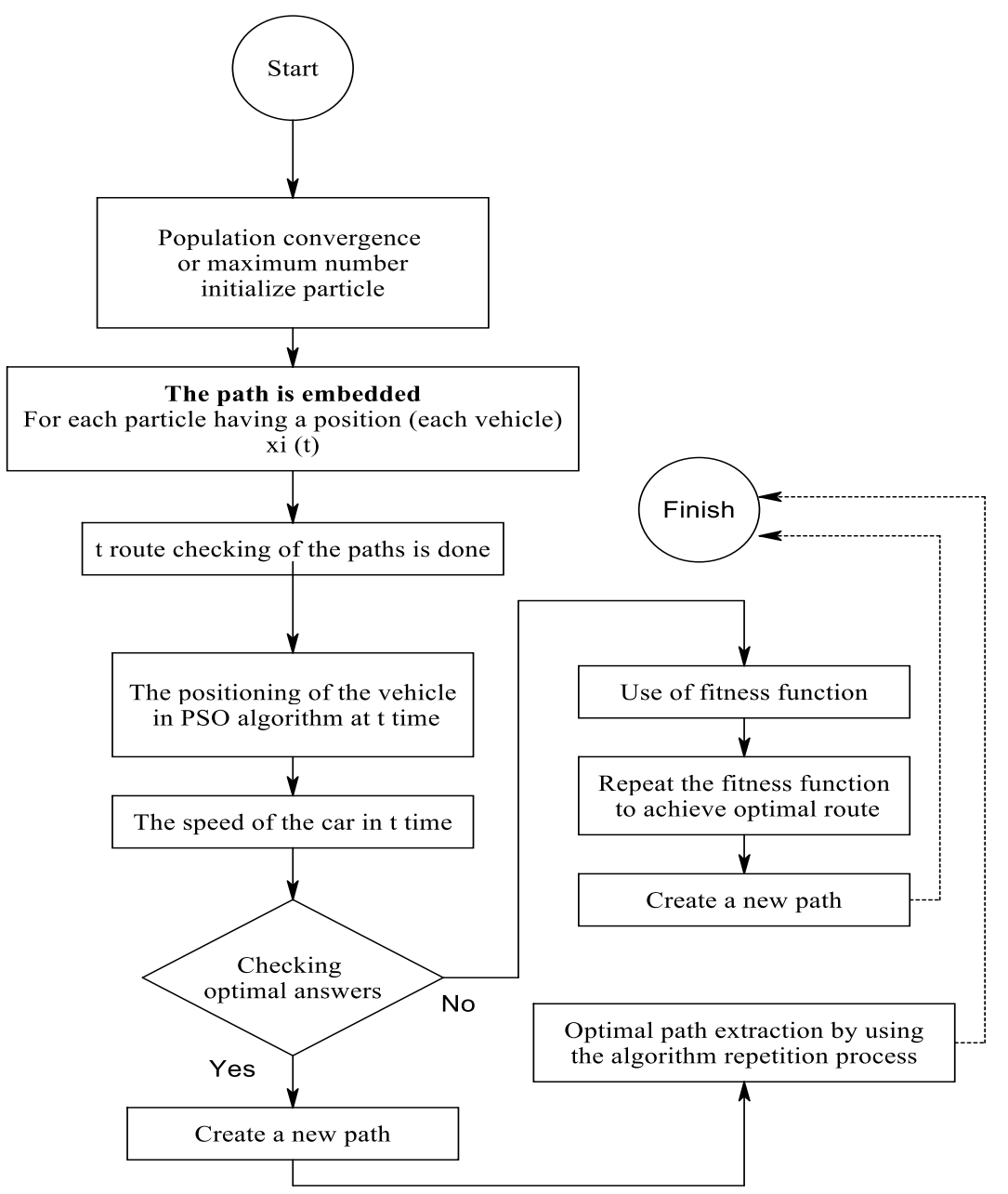

Fig. 3. Proposed path optimization algorithm by using collective particle optimization in VANET.

\section{Simulation and Evaluation}

We have used the MobiSim VANET package to implement the network model. The nodes in the network are randomly distributed for intended layers based on the class model. For RSUs, LTE with TDMA scheduling protocol is used. The UDP and TCP protocols are used to transmit data between nodes and clusters. Evaluation criteria for simulation are QoS related criteria such as Packer Delivery Rate (PDR), overhead, etc. Figure 4 represents the schematic overview on the simulation of this research. The XML file shown in Figure 4 has relative information to the vehicles schematic diagram. Also, NS2 simulator help on executing TCP, UDP and routing algorithms for wired and wireless networks. It is also possible with NS2 to change and define network algorithms and the vehicle's corresponding behavior. NS2 is implemented in a platform using $\mathrm{C}++$ programming language with an Otcl interpreter as user interface. NS implements various network protocols such as UDP and TCP, various traffic models such as $\mathrm{CBR}^{1}$, Web, Telnet, $\mathrm{VBR}^{2}$ and $\mathrm{FTP}^{3}$ routing algorithms as well as various queue management mechanisms such as RED, $\mathrm{CBQ}^{4}$ and Drop Tail.

\footnotetext{
${ }^{1}$ Constant bit rate

${ }^{2}$ Variable bit rate

${ }^{3}$ File Transfer Protocol

${ }^{4}$ Class-based queuing
} 


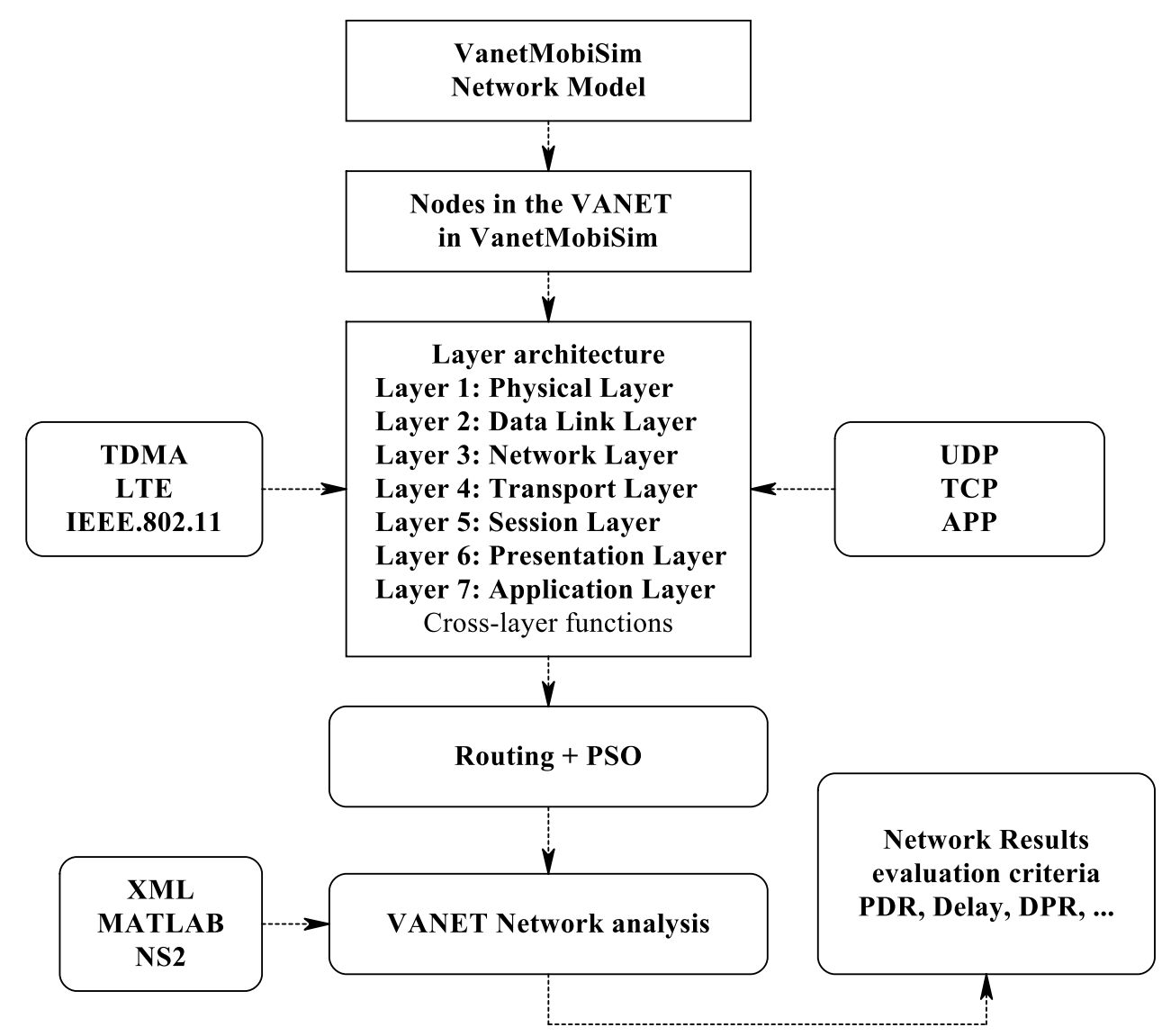

Fig. 4. Schematic overview of routing simulator in this research based on different transmission protocols.

In order to simulate and evaluate the proposed protocol, we need to choose a suitable mobility model for VANETSs. Each mobility model defines the movement pattern of the nodes including the pattern of displacement, velocity, and acceleration of the nodes over time. The more the mobility model in the simulation is in line with the suitable mobility model of moving nodes in real situation, the closer the simulation results will be to the reality. Figure 5 shows the classification of existing mobility models [23]:

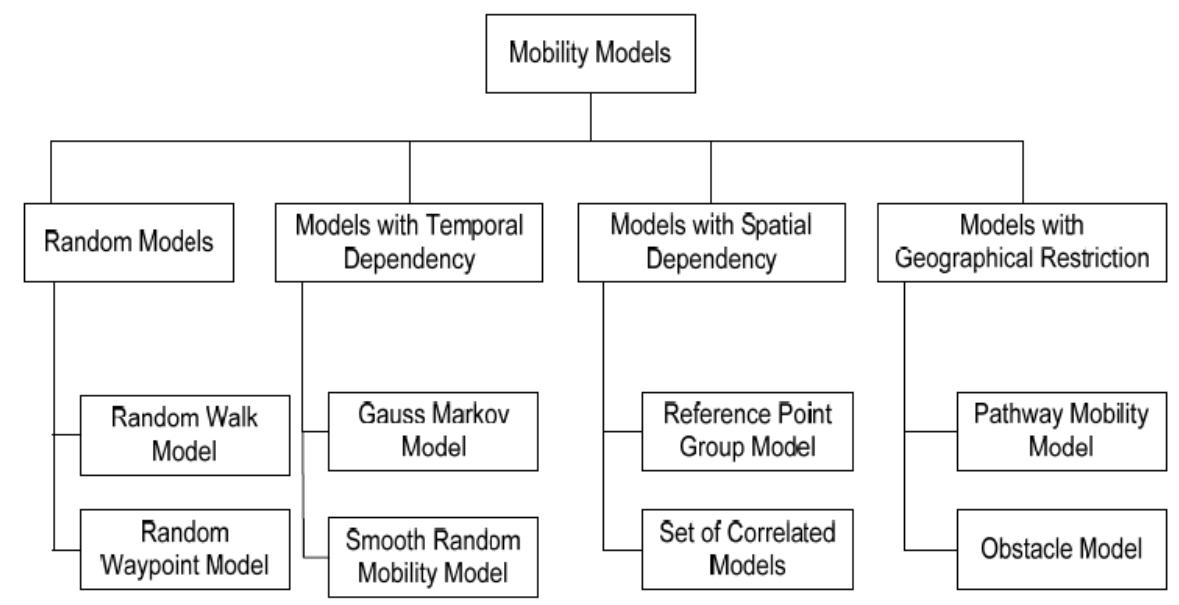

Fig. 5: Some of self-contained mobility for QoSR [39]. 
The Random Waypoint model was first presented and used to calculate the QoSR in self-contained networks. This model, each node stops for a predefined time called the pause time (PT), and then moves toward a random point in a straight line with a random velocity. After reaching the destination, it stops again for PT and repeats the same procedure to move to other points. CMU Monarch research team have developed the software tool to generate the Random Waypoint mobility model for NS2. By using this tool, user can define pause time, maximum speed, number of nodes, and dimensions of the node mobility environment as well as the duration of the network simulation.

The proposed algorithm is compared in different scenarios in terms of different evaluation criteria (permittivity and delay), with SAMQ algorithm [4]. Our simulator is a combination of NS2 software with VanetMobiSim.to simulate vehicular ad hoc networks. NS2 is a discrete-event simulation tool suitable for dynamic nature of communication networks[24], [25]. VanetMobiSim simulates roads and geo-routing of transport in urban space scenarios in VANETs. We use random mobility to simulate and evaluate proposed protocol for VANETs. The more simulation parameters are in line with mobility pattern of vehicles in reality, the results are more accurate and reliable. Nodes stop at a predefined time (stop time), move to a randomly determined point with a random speed. Throughput is calculated according to Equation (8). In this relation $\mu$ the number of bits received in $\mathrm{t}$ time unit. The EndToEnd delay is calculated according to equation (9). D is delay of a packet, $T_{d}$ is the arrival time of packet to destination, and $T_{s}$ is transportation time. Packet delivery ratio (PDR) is equal to ratio of received packets to (Receive_Pckt) number of sent ones (Sent_Pckt) and is presented in equation (10).

Throughput $=\frac{\mu}{t}$

$\mathrm{D}=\mathrm{T}_{\mathrm{d}}-\mathrm{T}_{\mathrm{s}}$

PDR $=$ Received Packets $/$ Sent Packets

\subsection{Evaluation}

The results of proposed method (QoSR-PSO) created on collective PSO is presented on a 10-km six-lane traffic scenario with two independent driving directions for vehicles. We implement the highway with typical mobility presented in [51], which is based on road traffic concept, instructions and drivers' behavior. The results are compared with SAMQ, GA, MPLS, VACO, PUMA and Multi-hop methods for different criteria; packet delivery rates, routing overhead, delay and lost packets [4]. Figure 4 illustrates simulation scheme is shown in Linux urban environment [4]. Table 3 contains simulation parameters in NS2. 


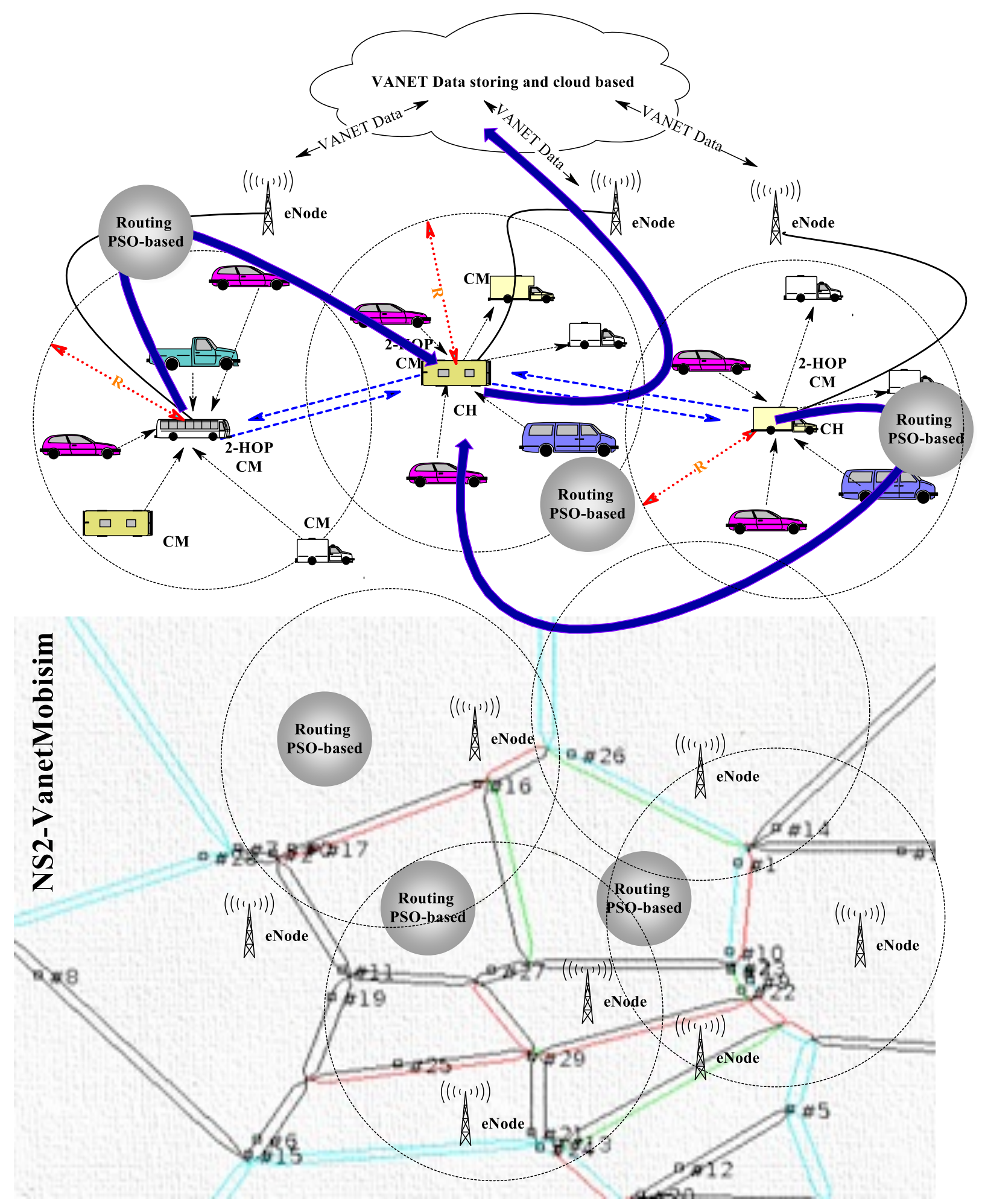

Fig. 4. simulation scheme in Linux environment. 
Table 3 - Simulation parameters in NS2.

\begin{tabular}{|l|l|}
\hline \multicolumn{1}{|c|}{ Parameters } & \multicolumn{1}{c|}{ Amounts } \\
\hline Node movement model & Random Waypoint \\
\hline The size of network & 25 vehicle \\
\hline number of Interest & interest for each simulation run \\
\hline Speed of vehicles & $10-250 \mathrm{~km} / \mathrm{h}$ \\
\hline Mobility model & Random mobility model \\
\hline The transmission radius of vehicles & $150 \mathrm{~m}$ \\
\hline Along the highway & From 1 to $4 \mathrm{~km}$ \\
\hline Simulation Area & $1 \mathrm{~km} \times 10 \mathrm{~km}$ \\
\hline MobilityModel & Highway Range \\
\hline CommunicationRange & $450-500$ (m) \\
\hline MACLayer & IEEE80211p \\
\hline Vehicles'Velocities & NormallyDistributed \\
\hline Vehicles'Distances & ExponentiallyDistributed \\
\hline NumberOfRuns (Num.) & $20,30,40,50$ \\
\hline SimulationDuration & $250-300$ seconds \\
\hline SAMQparameters[4]. & U0 $=0.6, \alpha=0.45, \beta=0.55, \tau 0=5, \mathrm{~N}=5$ \\
\hline StopTime (s) & $1 \mathrm{~s}$ \\
\hline
\end{tabular}

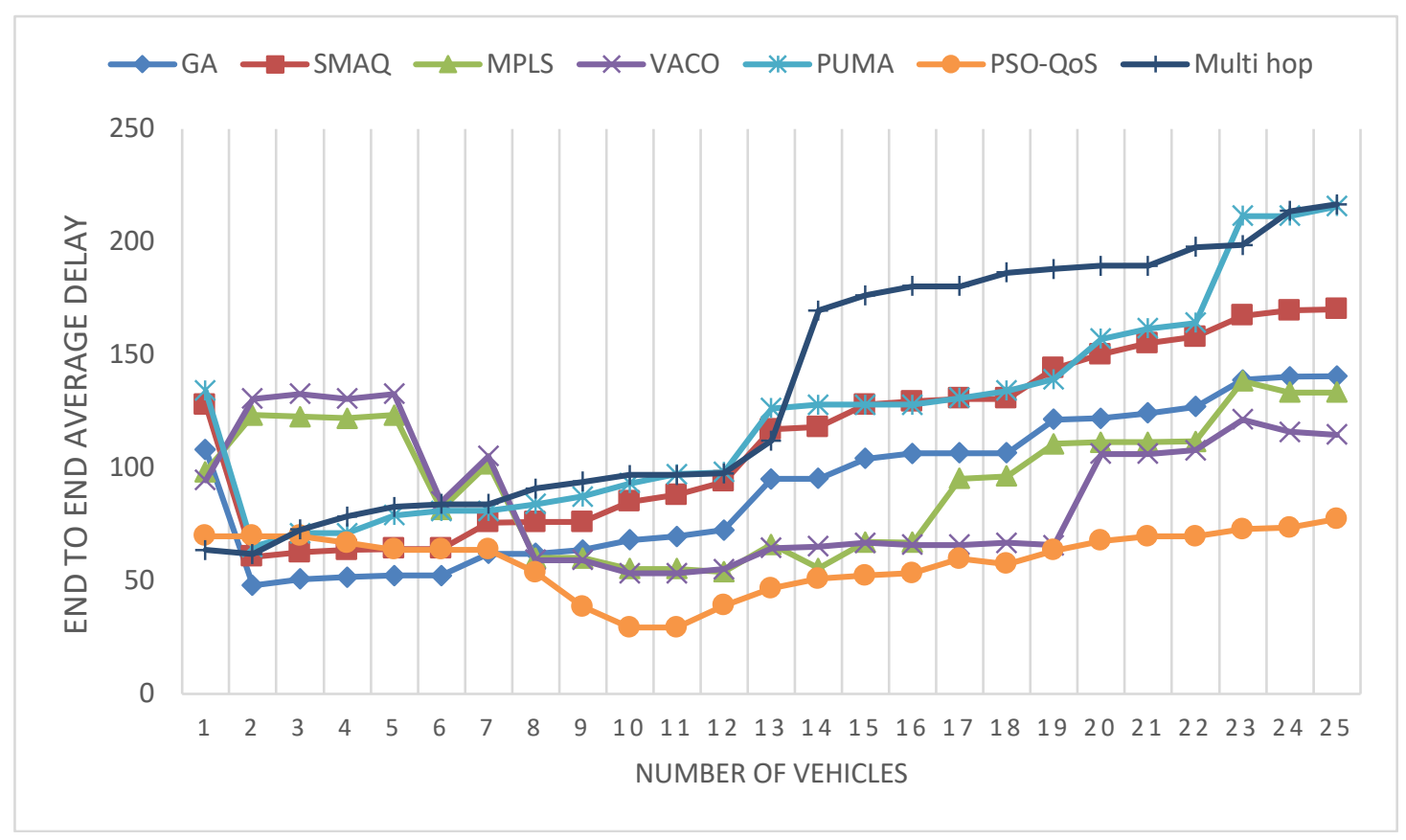

Fig. 5. The details of Average E2Ed delay transmission in VANETs.

\subsection{Simulation Results}

Our simulation is based on NS2 wireless package with the stop time of one second. We have compared our result with GA, SAMQ, MPLS, VACO, PUMA, Multi-hop for average EndtoEnd delay (E2Ed) (Figure 5), an average time for a packet to reach its destination is defined as packet delay. E2Ed is determined by stand in line delay, broadcasting, packaging and Sending. By increasing maximum speed, average E2Ed increases in all algorithms because of network topology and 
breakdown path rate. The proposed method has less delay compare to other methods due to the simulation environment and node distribution. The broken path causes high delay in SAMQ. The genetic algorithm does not provide adequate latency due to the high overhead and complex connections between RSUs and vehicles and the high vehicle environment.

In MPLS method, labeling in routing and updating of switches in the routing process produces high spatial complexity and as a result, delay will increase. In PUMA, due to high synchronization and mobility, the latency rate increases MPLS has large number of updates and switches synchronization and this is causing delay for labeling neighbors and adjacent nodes to form clusters. E2Ed delay in the QoSR-PSO is lower than other algorithms, smaller packets are transmitted in the network and nodes can easily obtain their requested packets. The QoSR-PSO has 17\% lower than SAMQ algorithm in E2Ed. Figure 5 demonstrates the positive relation between the average PDR and Num.Of Vehicles. Due to the high complexity of VACO algorithm, the delivery rate and is not optimal. In SAMQ and the QoSR-PSO, the packet delivery rate is increasing by adding nodes to the simulation environment. By rising the Num.of nodes and VANET density, E2Ed in all algorithms is reduced, which is due to increasing number of routes among Sender and destination. The maximum speed of nodes is 130 $\mathrm{m} / \mathrm{s}$ and the number of nodes is 25 . Using network coding to transfer packets affect stop time of the nodes. By rising the Num.of nodes in area, network dynamics decreases and the network becomes more constant, which does not lead to rerouting. That's the reason that our algorithm works more efficiently. Also link loss ratio is lower, resulting in less delay. Delay has been decreased by 8.4, 10.2, 7.1, 4.4, 11.8 and 15.6 percent for GA, SAMQ, MPLS, VACO, PUMA and multi hop respectively.

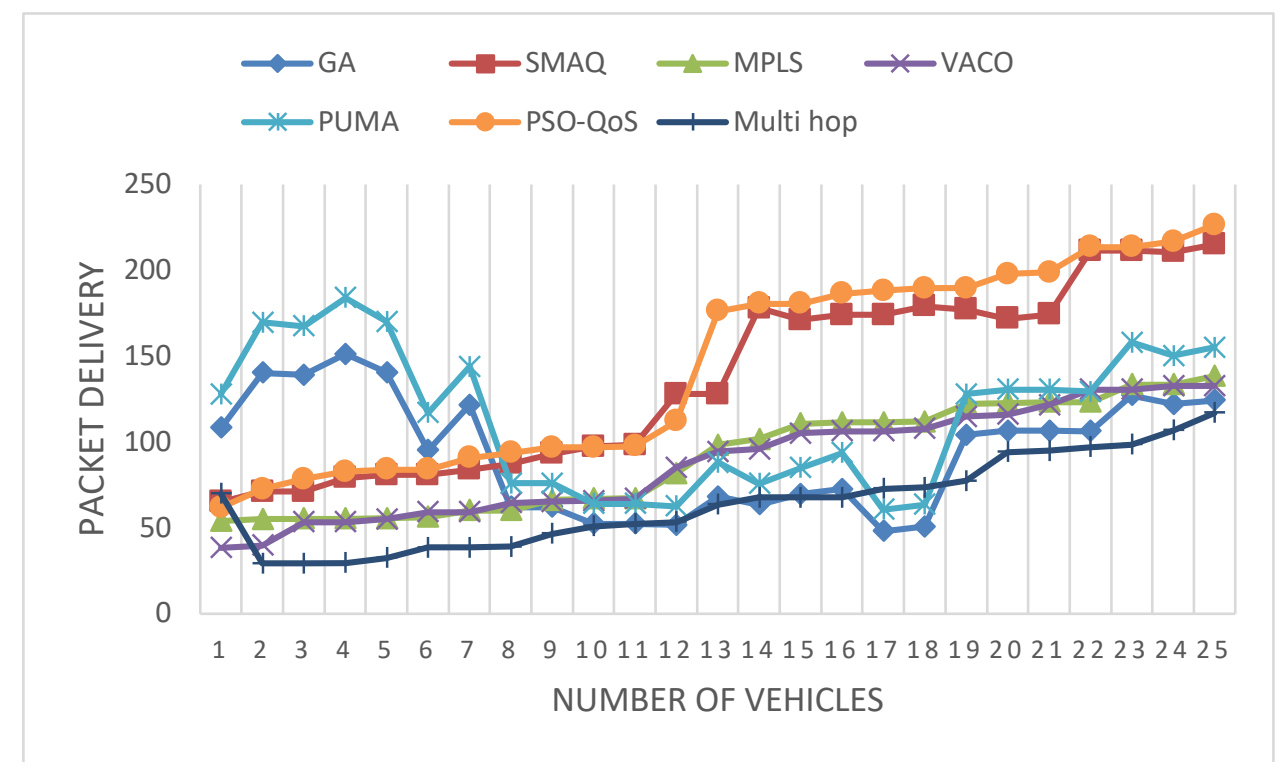

Fig. 6. The Details of PDR for Number of vehicles and Data Transmission in VANETs.

Results on comparing average packet delivery rate is compared with benchmark studies in figure 6 . Higher delivery rate in our presented method is because of proper positioning of the particles and clustering. By increasing speed and the number of in simulation scenario, SAMQ and MPLS deliver less number of packets. Packet delivery in GA and PUMA methods also decrease because of the spatial complexity of these methods and the overhead in the network which reduces the throughput and packet delivery rate. SAMQ has the closest performance to our QoSR-PSO method. Proposed method improves packet delivery rate compare to GA 13\%; SAMQ 4.3 \%, MPLS 10.2\%; VACO 11\%; PUMA 16\%; and 18\% for multi-hop.

In Figure 7 control overhead is evaluated based on the number of nodes. As shown in Figure, control overhead increases in the QoSR-PSO. The simulations are based on the frequency of repetitions between sender and receiver. Movement of packets and the initial expectation for the formation of the first hub for routing vehicles cause high control overhead to the network. High generated overhead at the beginning of the simulation shows that the formation of long-delayed hubs raise the complexity of VANETs, and this complexity increases as network E2Ed. As the V2V and V2RSU communication increases by executing the simulation for longer time, control overload becomes optimum. The high complexity in PUMA generates high overload. In metaheuristic methods such as SMAQ and GA, the overhead method is improving due to the number of simulation iterations. The QoSR-PSO improves control overload compared to GA 11.6\%; SAMQ 5.7\%; MPLS 5.2\%; VACO, $3.1 \%$; PUMA 10.1\% and Multi-hop for 7.2\%. 


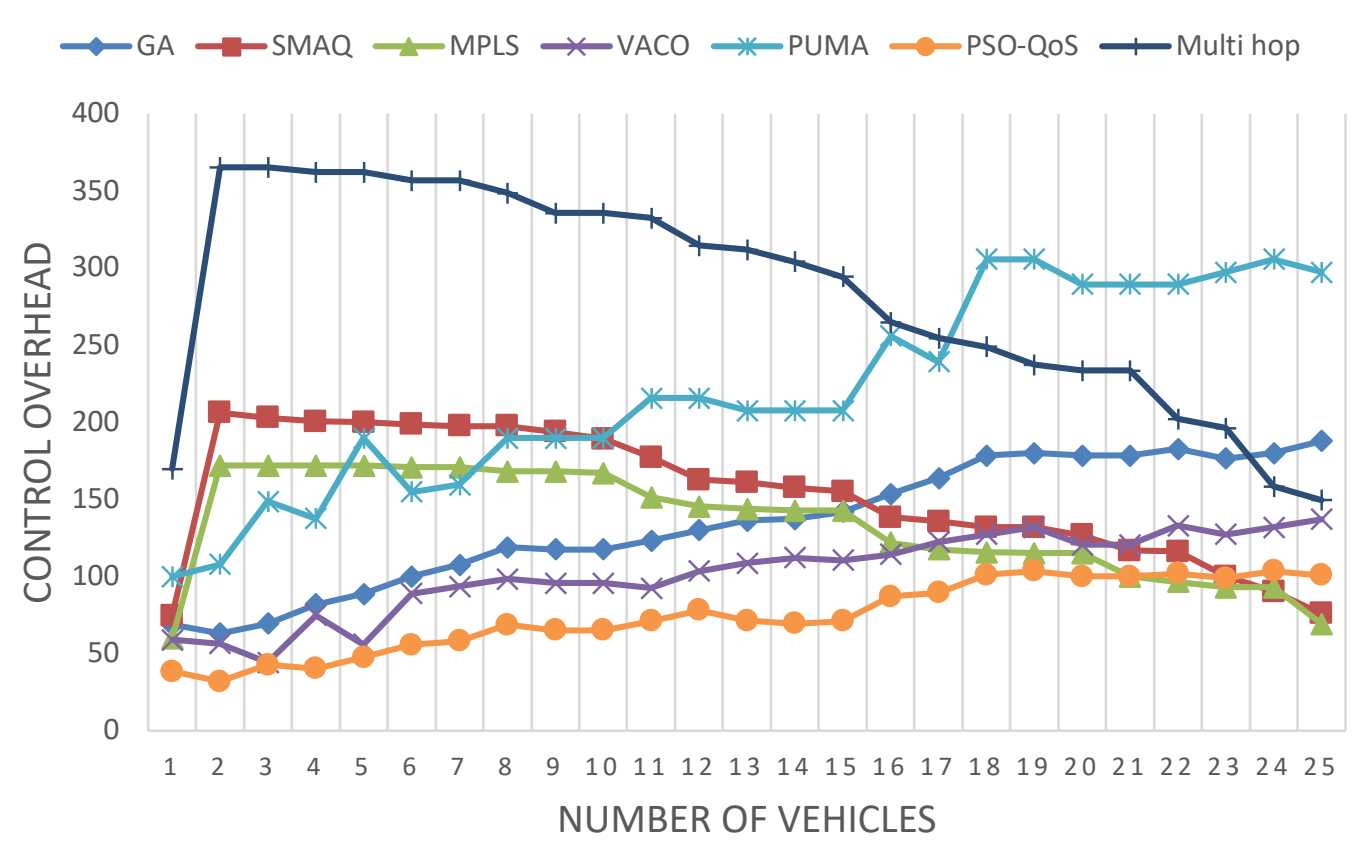

Fig. 7. A: The details of Overhead and Data transmissions in VANETs

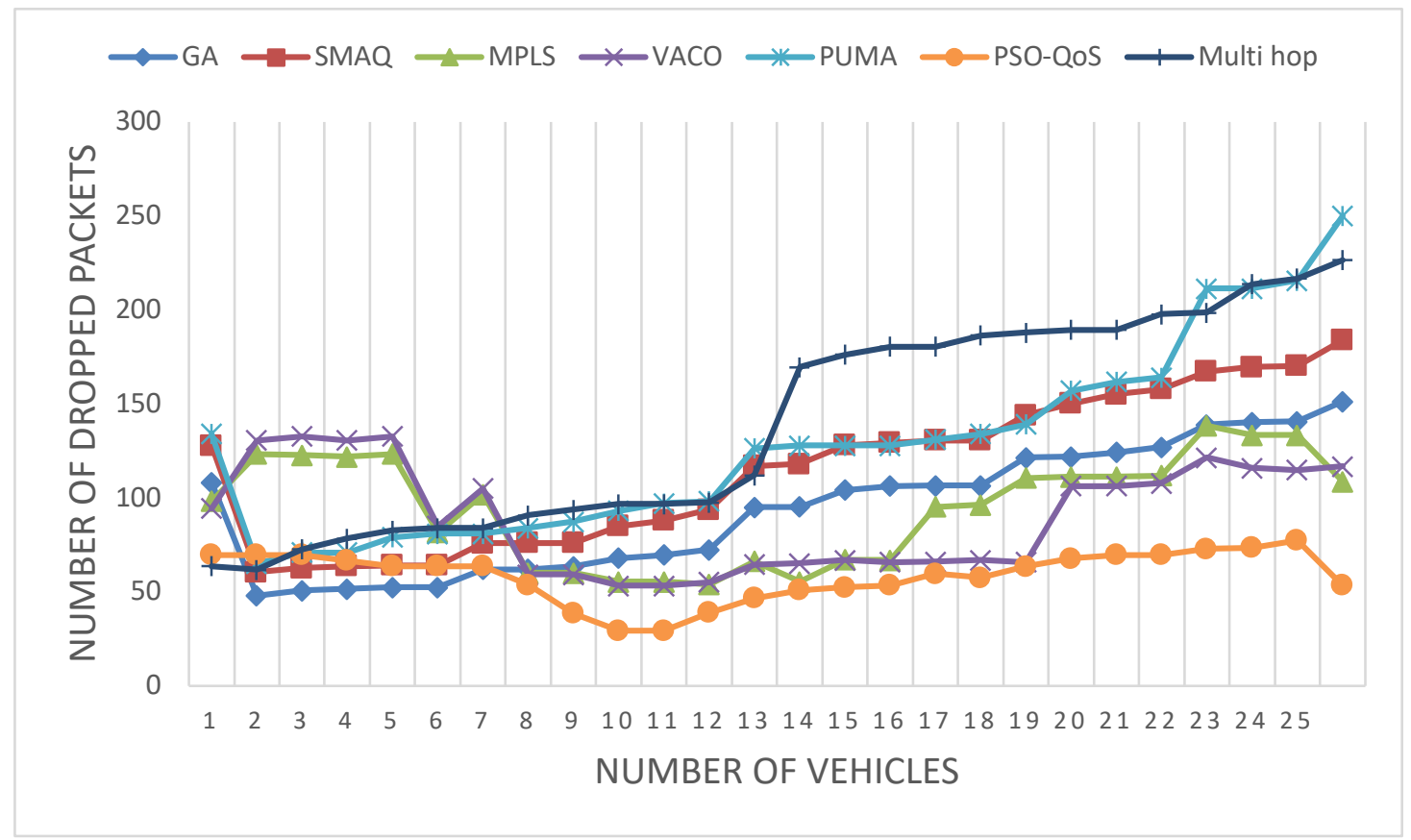

Fig. 8. Dropped packets rate for Number of vehicles

Figure 8 considers dropped packets rate. Increasing the nodes does not affect in QoS and dropped packets because in the simulations with PSO the corresponding output is optimized and ultimately the Num.of packets is reduced. In VACO, Based on high path complexity generation and number of lost packets is high. SMAQ and GA behave similarly for packet loss. SAMQ like the QoSR-PSO has performed very well in establishing the received packets and has a low rate of lost packets. High complexity of GA has caused many packets to be lost. Sending packets between several different hubs and updating the created paths in multi-hop has increased the packet loss rate. On PDR it can be seen that the missing packets have increased. Spatial complexity of MPLS and high synchronization and mobility of PUMA are other reasons of high dropped packets rate. The proposed method compared to the GA 10.4\%; SAMQ 14.8\%; MPLS 8.7\%; VACO 7.8\%; PUMA 14.5\%; Multi-hop 11.6\% improves the packet loss rate. 


\section{Conclusion}

Rapid advancements VANET have made a lot of changes in various areas in our everyday lives such as safety in roads. Mobile ad hoc networks have no previous defined topology. Nodes can move freely in these networks and the topology changes momentarily. Due to the mobility of nodes in VANETs, the information transmission is an important issue because it informs other vehicles about traffic or accident situations. PSO by modeling the behavior of a set of particles looks for the optimal solution. NS2 open source environment and VANET-MOBISIM software are used to simulate our QoSR-PSO. We have evaluated our presented approach for delay, dropped packet rate, control overhead and Average packet delivery rate by increasing the vehicles. The Max of vehicles are 25 with the max speed of 150 meter per second. Delay has been decreased by $8.4,10.2,7.1,4.4,11.8$ and 15.6 percent for GA, SAMQ, MPLS, VACO, PUMA and multi hop respectively. Packet delivery has improved 13\% for GA; $4.3 \%$ for SAMQ, $10.2 \%$ for MPLS; $11 \%$ for VACO; $16 \%$; for PUMA and $18 \%$ for multi-hop. Similarly control overload is improved compared to GA11.6\%; SAMQ 5.7\%; MPLS 5.2\%; VACO, 3.1 \%; PUMA $10.1 \%$ and Multi-hop for 7.2\%. Finally, packet loss by our presented approach is improved compare to GA $10.4 \%$; SAMQ 14.8\%; MPLS 8.7\%; VACO 7.8\%; PUMA 14.5\%; Multi-hop 11.6\%.

\section{Compliance with ethical standards}

\section{Conflict of interest}

The author declares that he has no conflicts of interest.

\section{Ethical approval}

This article does not contain any studies with human participants or animals performed by any of the authors.

\section{Research involving human participants and/or animals}

This article does not contain any studies with human participants performed by any of the authors.

\section{Informed consent}

Informed consent was obtained from all individual participants included in the study.

\section{References}

[1] C. Wu, S. Ohzahata, and T. Kato, "Data dissemination with dynamic backbone selection in vehicular ad hoc networks," IEEE Veh. Technol. Conf., 2013.

[2] W. Liang, Z. Li, H. Zhang, S. Wang, and R. Bie, "Vehicular Ad Hoc Networks : Architectures, Research Issues, Methodologies , Challenges , and Trends," vol. 2015, 2015.

[3] J. Kponyo, Y. Kuang, E. Zhang, and K. Domenic, "VANET cluster-on-demand minimum spanning tree (MST) Prim clustering algorithm." 2013.

[4] M. Hashem Eiza, T. Owens, Q. Ni, and Q. Shi, "Situation-Aware QoS Routing Algorithm for Vehicular Ad hoc Networks," IEEE Trans. Veh. Technol., vol. 9545, no. c, pp. 1-1, 2015.

[5] M. Hashem Eiza, T. Owens, and Q. Ni, "Secure and Robust Multi-Constrained QoS aware Routing Algorithm for VANETs," IEEE Trans. Dependable Secur. Comput., pp. 1-1, 2015.

[6] E. C. Eze, S. Zhang, and E. Liu, "Vehicular ad hoc networks (VANETs): Current state, challenges, potentials and way forward," Automation and Computing (ICAC), 2014 20th International Conference on. pp. 176-181, 2014.

[7] G. Wolny, "Modified DMAC Clustering Algorithm for VANETs," in Systems and Networks Communications, 2008. ICSNC '08. 3rd International Conference on, 2008, pp. 268-273.

[8] K. Abboud, S. Member, and W. Zhuang, "Stochastic Modeling of Single-Hop Cluster Stability in Vehicular Ad Hoc Networks," ACM MSWiM, vol. 9545, no. c, pp. 65-72, 2015.

[9] M. K. Hossain, S. Datta, S. I. Hossain, and J. Edmonds, "ResVMAC: A Novel Medium Access Control Protocol for Vehicular Ad hoc Networks," Procedia Comput. Sci., vol. 109, pp. 432-439, 2017.

[10] M. Sohail and L. Wang, "3VSR: Three valued secure routing for vehicular ad hoc networks using sensing logic in adversarial environment," Sensors (Switzerland), vol. 18, no. 3, pp. 1-24, 2018.

[11] R. Bala and C. R. Krishna, "Scenario based performance analysis of AODV and GPSR routing protocols in a VANET," Proc. 2015 IEEE Int. Conf. Comput. Intell. Commun. Technol. CICT 2015, pp. 432-437, 2015.

[12] M. Hadded et al., "Ad Hoc Networks To cite this version : A Novel Angle-based Clustering Algorithm for Vehicular Ad Hoc 
Networks," 2016.

[13] Z. Lei, L. Tao, L. Wei, Z. Siting, and L. Jianfeng, "Cooperative spectrum allocation with QoS support in cognitive cooperative vehicular ad hoc networks," Communications, China, vol. 11, no. 10. pp. 49-59, 2014.

[14] D. Perdana, M. Nanda, R. Ode, and R. F. Sari, "Performance evaluation of PUMA routing protocol for Manhattan mobility model on vehicular ad-hoc network," Telecommunications (ICT), 2015 22nd International Conference on. pp. 80-84, 2015.

[15] R. Monteiro, S. Sargento, W. Viriyasitavat, and O. K. Tonguz, "Improving VANET protocols via network science," Vehicular Networking Conference (VNC), 2012 IEEE. pp. 17-24, 2012.

[16] P. Salvo, F. Cuomo, A. Baiocchi, and I. Rubin, "Investigating \{VANET\} dissemination protocols performance under high throughput conditions," Veh. Commun., vol. 2, no. 4, pp. 185-194, 2015.

[17] M. Watfa, Advances in Vehicular Ad-Hoc Networks: Developments and Challenges: Developments and Challenges. IGI Global, 2010.

[18] L. Zhang and H. El-Sayed, “A Novel Cluster-Based Protocol for Topology Discovery in Vehicular Ad Hoc Network,” Procedia Comput. Sci., vol. 10, pp. 525-534, 2012.

[19] C. . Palazzi, "An inter-vehicular communication architecture for safety and entertainment," IEEE Trans. Intell. Transp. Syst., 2010.

[20] S. A. M. Ahmed, S. H. S. Ariffin, and N. Fisal, "Network coding techniques for VANET advertising applications," EURASIP J. Wirel. Commun. Netw., vol. 2015, no. 1, p. 200, 2015.

[21] S. Ucar, S. C. Ergen, and O. Ozkasap, "Multihop-Cluster-Based IEEE 802.11p and LTE Hybrid Architecture for VANET Safety Message Dissemination," IEEE Trans. Veh. Technol., vol. 65, no. 4, pp. 2621-2636, 2016.

[22] B. R, K. Gupta, and S. B. S., "Performance evaluation of topology based routing protocols for VANETs in urban scenarios," in Fourth International Conference on Advances in Recent Technologies in Communication and Computing (ARTCom2012), 2012, pp. 35-37.

[23] M. P. Ananda and R. Devaraju, "Implementation of Enhanced New Stable Election Protocol- ENHSEP in NS2 Platform," Int. Res. J. Eng. Technol., vol. 4, no. 7, pp. 970-979, 2017.

[24] N. Mirjazaee and N. Moghim, "An opportunistic routing based on symmetrical traffic distribution in vehicular networks," Comput. Electr. Eng., vol. 47, pp. 1-12, 2015.

[25] M. Fathy, S. Gholamalitabarfirouzjaee, and K. Raahemifar, "Improving QoS in VANET Using MPLS a," vol. 10, pp. 1018$1025,2012$. 
Figures

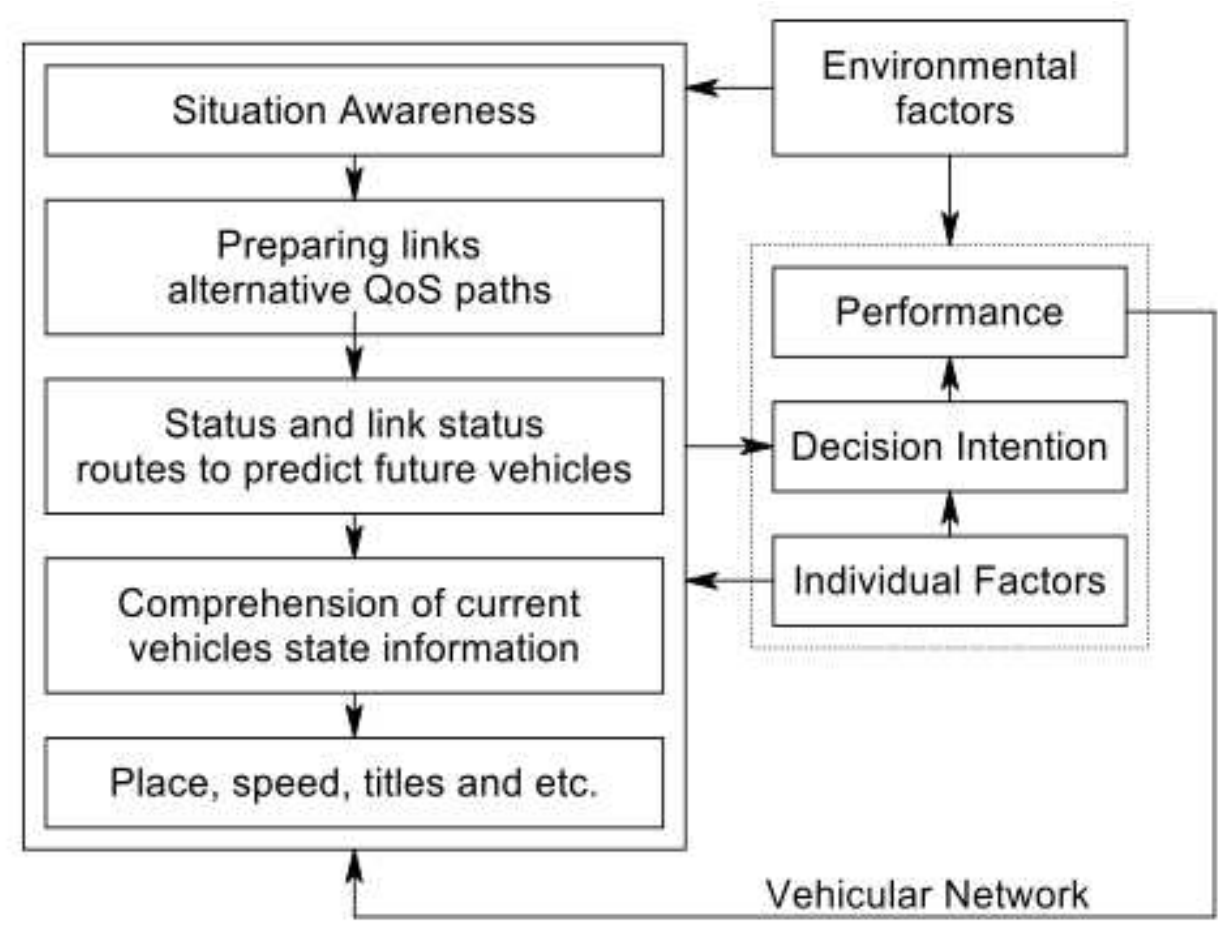

Figure 1

Classical model for Routing in VANETs [4]. 


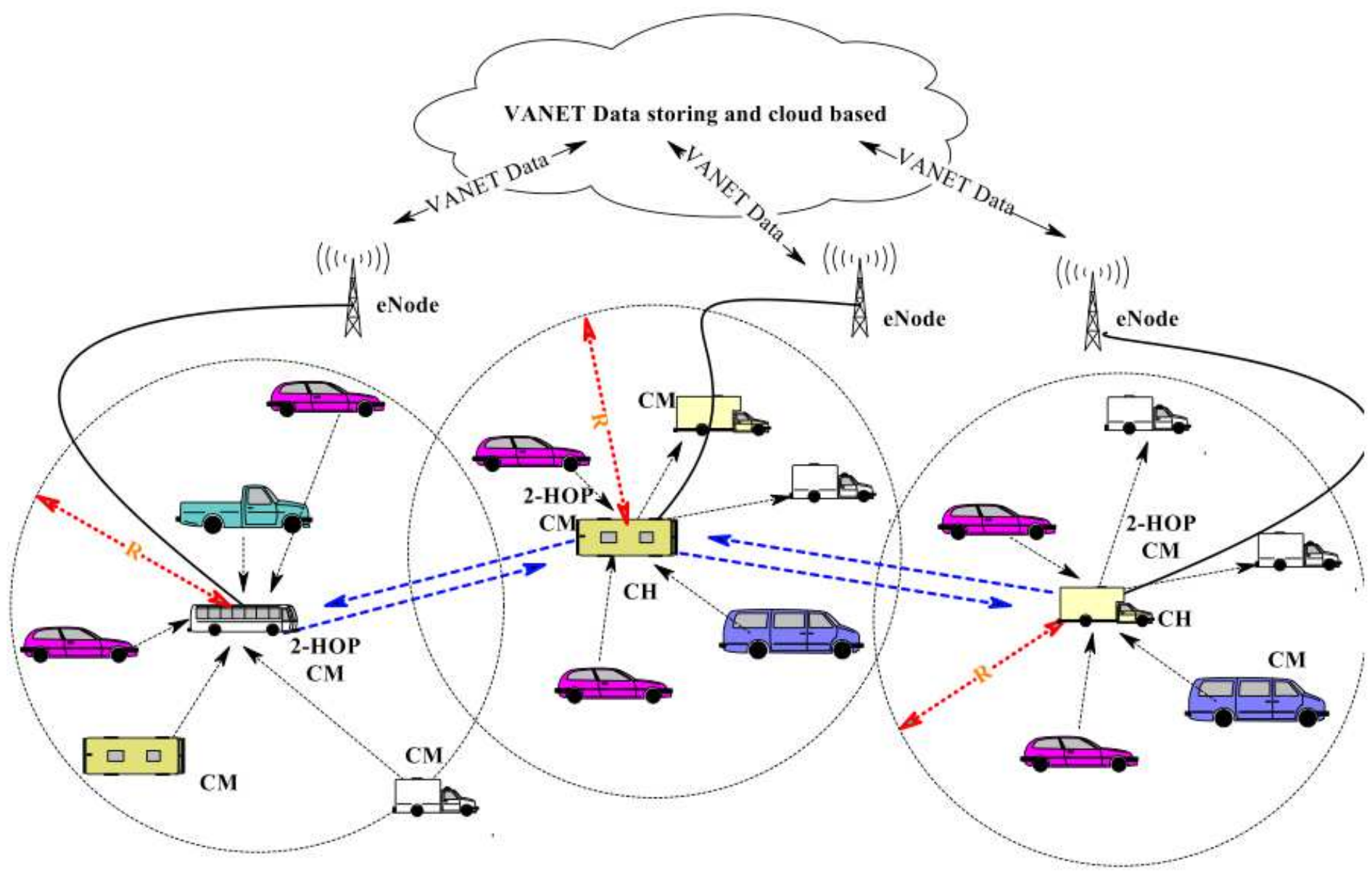

Figure 2

An overview of node communication in VANETs[21]. 


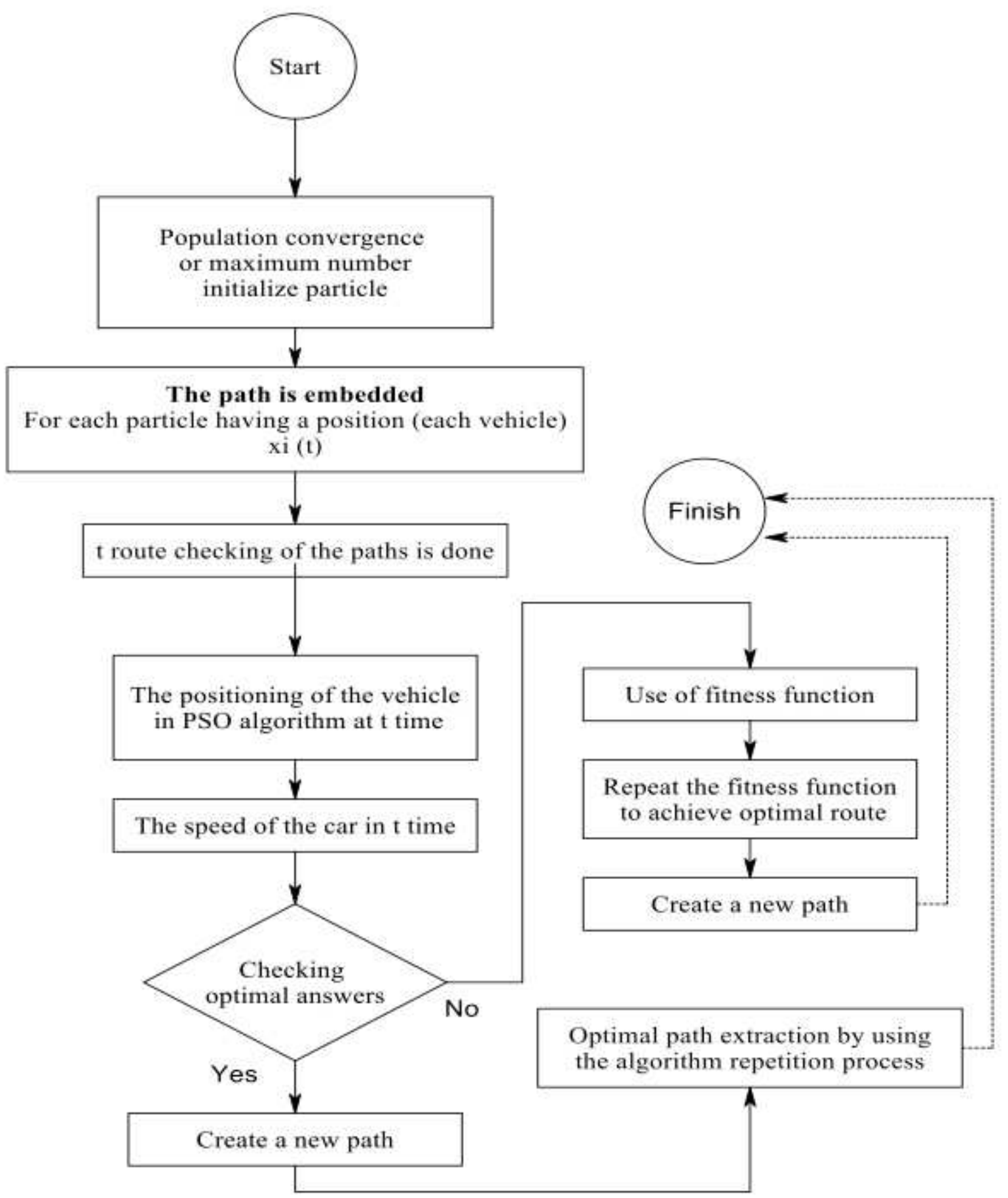

Figure 3

Proposed path optimization algorithm by using collective particle optimization in VANET. 


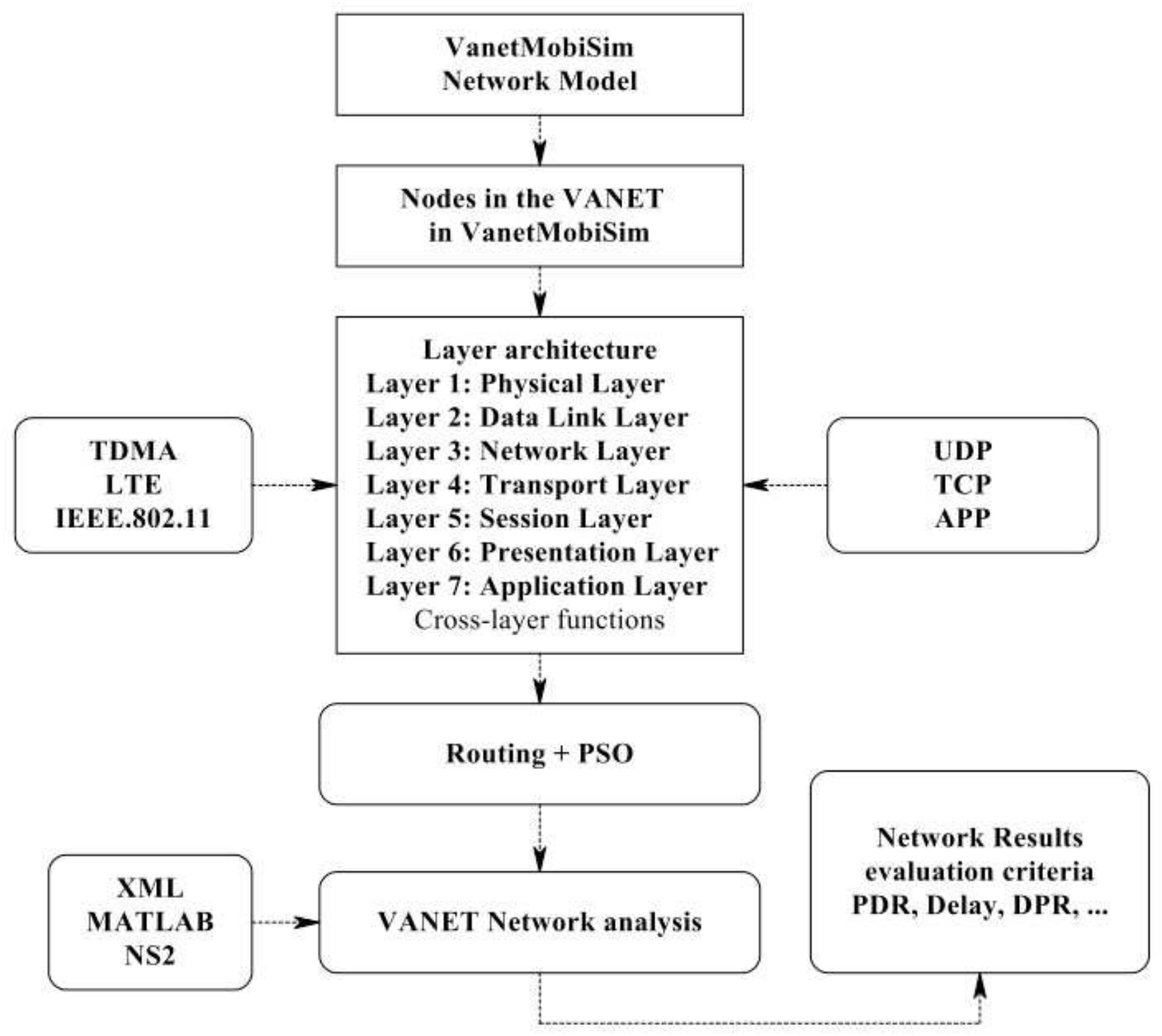

Figure 4

Schematic overview of routing simulator in this research based on different transmission protocols. 


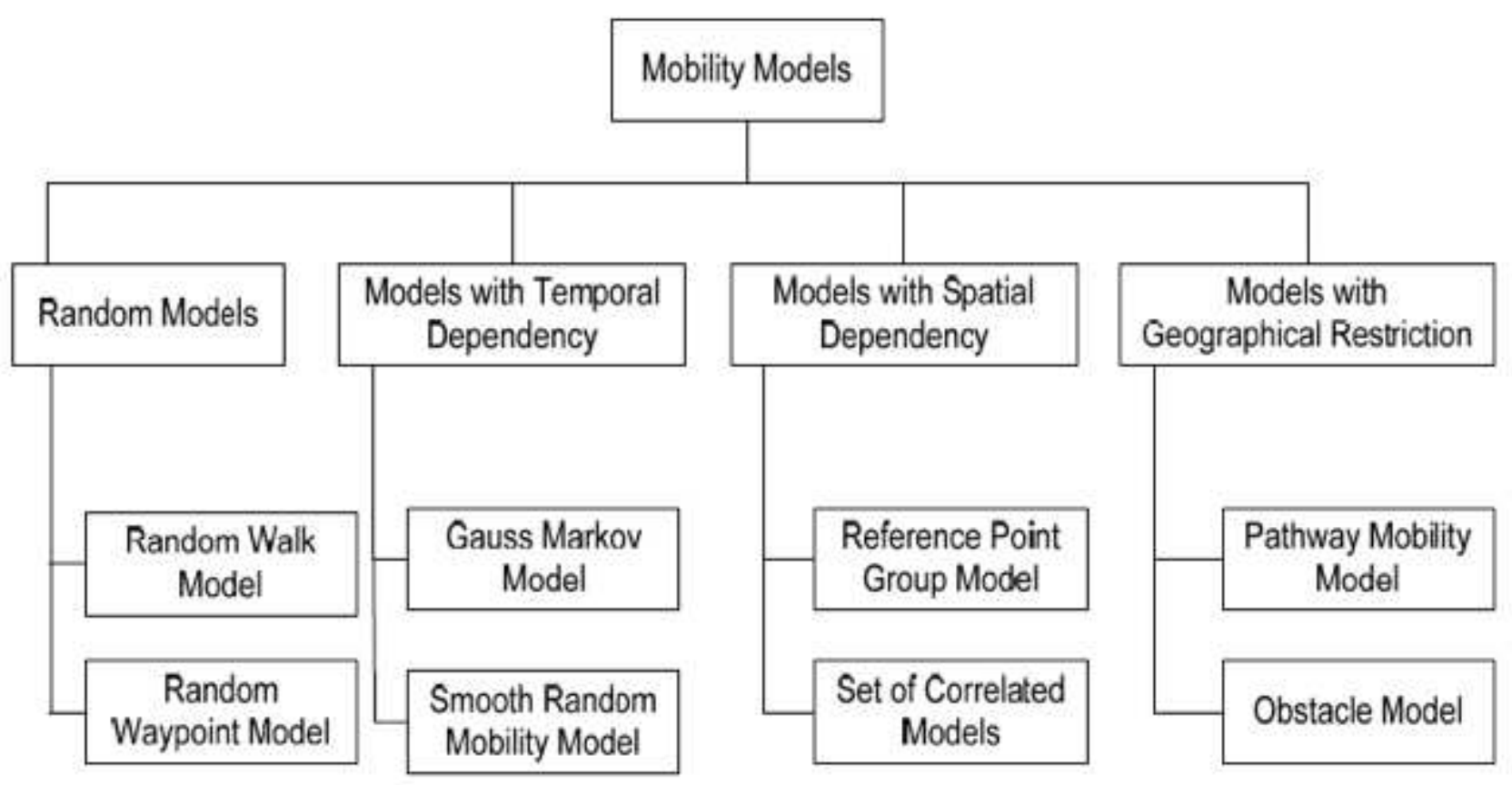

\section{Figure 5}

Some of self-contained mobility for QoSR [39]. 


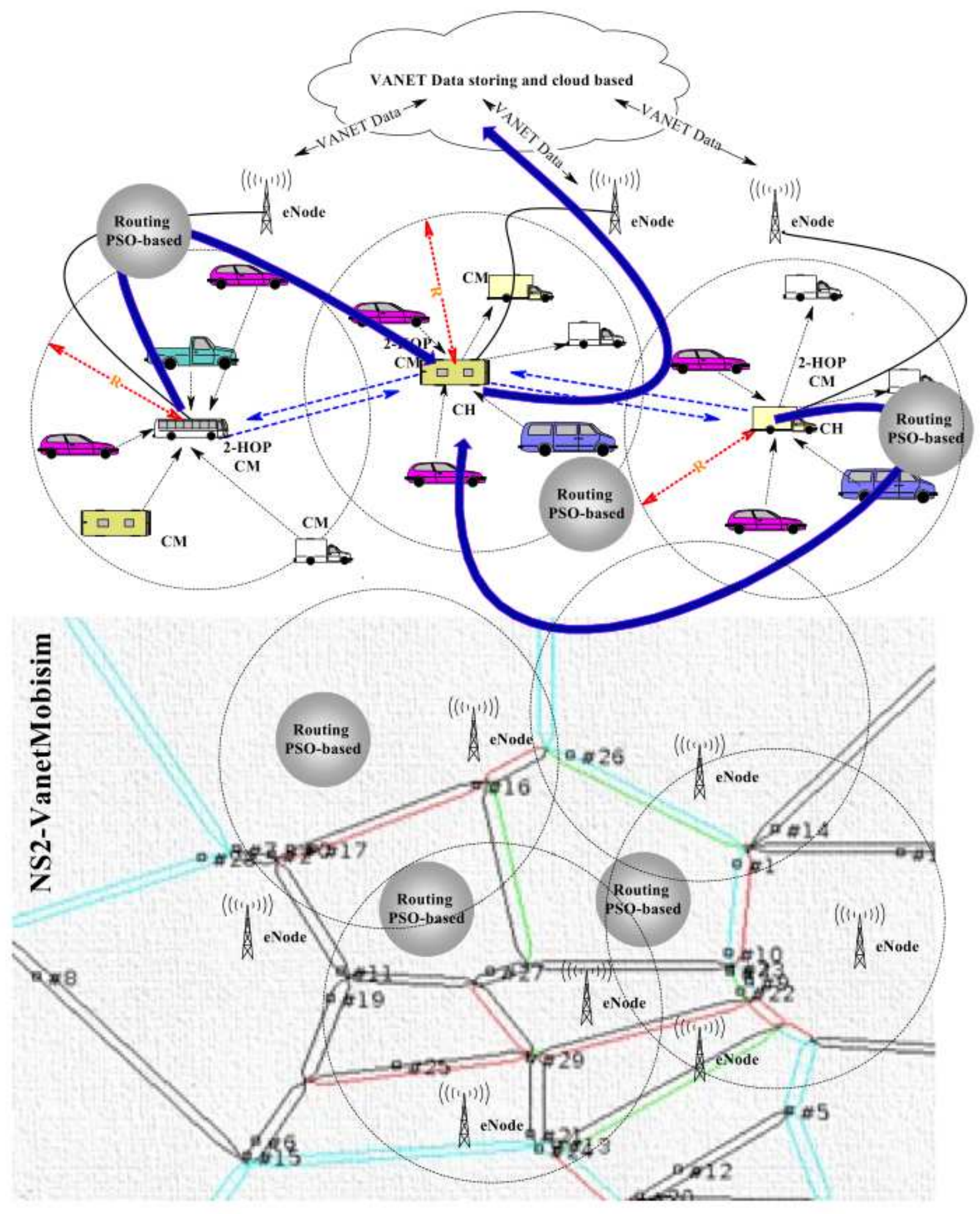

Figure 6

simulation scheme in Linux environment. 


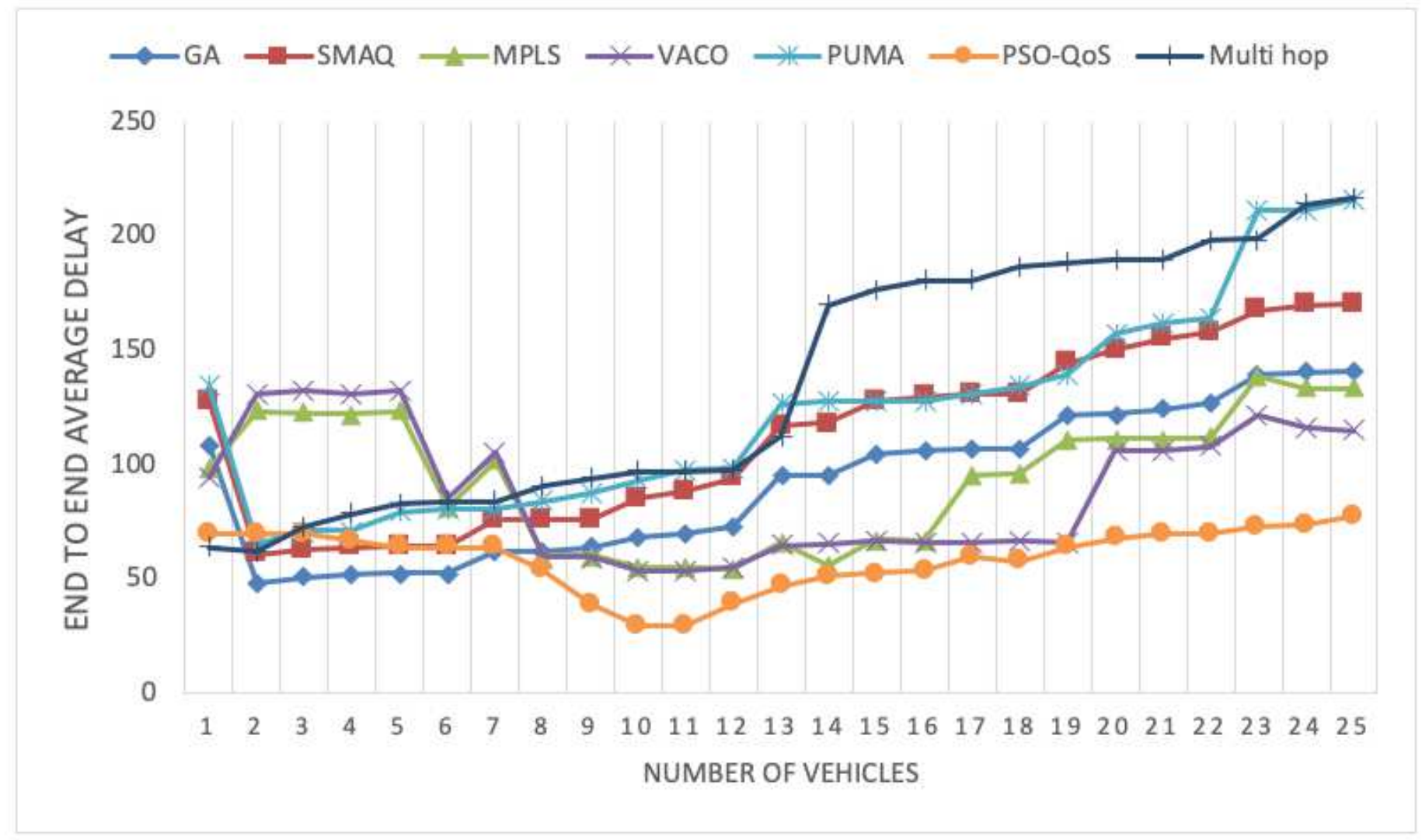

Figure 7

The details of Average E2Ed delay transmission in VANETs. 


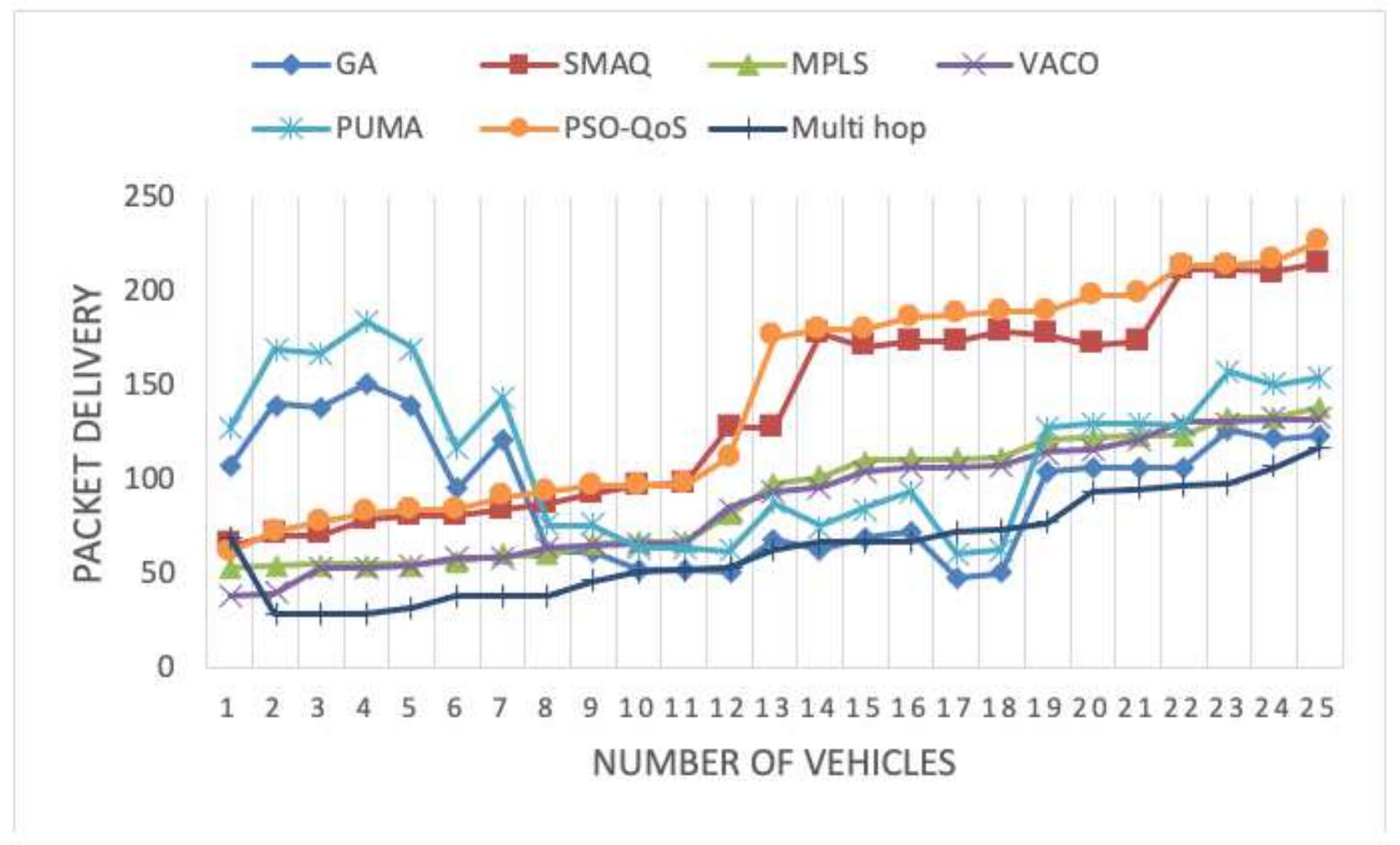

Figure 8

The Details of PDR for Number of vehicles and Data Transmission in VANETs. 


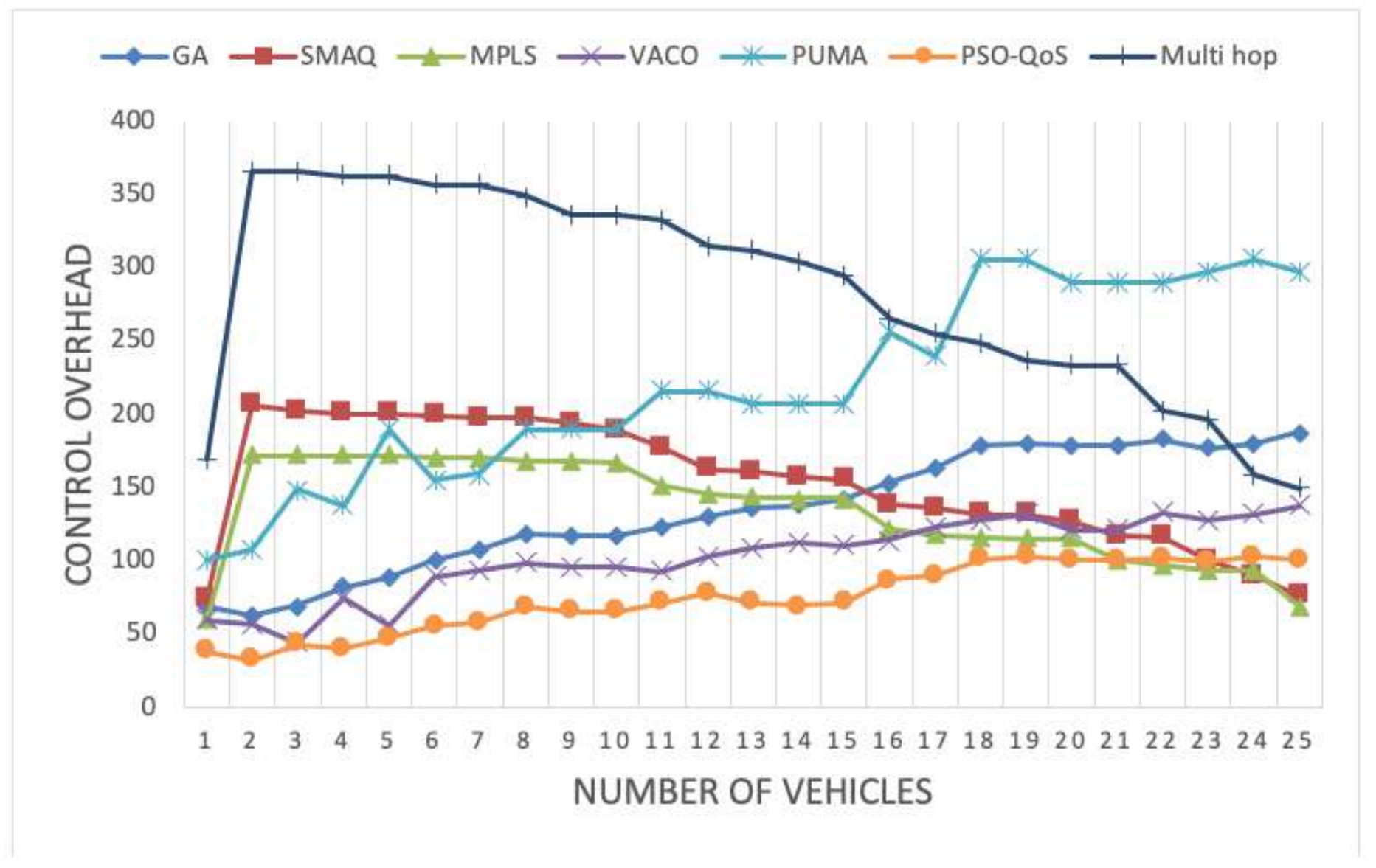

Figure 9

A: The details of Overhead and Data transmissions in VANETs 


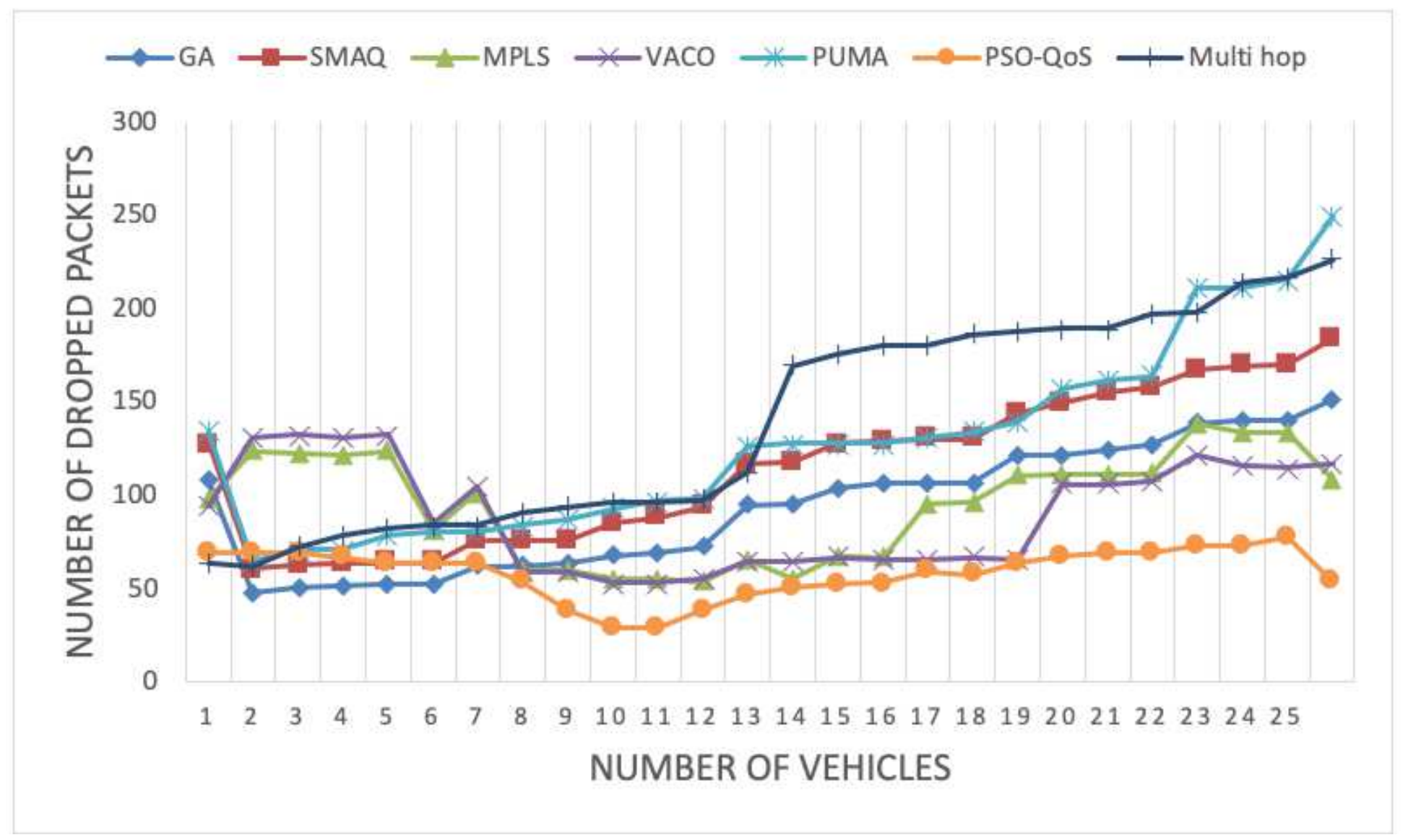

Figure 10

Dropped packets rate for Number of vehicles 